\title{
A NEW LARGE SUPER-FAST ROTATOR: (335433) 2005 UW163
}

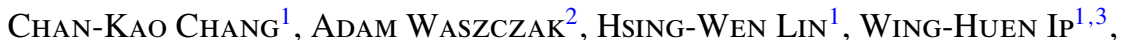 \\ Thomas A. Prince ${ }^{4}$, Shrinivas R. KulKarni ${ }^{4}$, Russ Laher ${ }^{5}$, AND Jason Surace ${ }^{5}$ \\ ${ }^{1}$ Institute of Astronomy, National Central University, Jhongli, Taiwan; rex@ astro.ncu.edu.tw \\ ${ }^{2}$ Division of Geological and Planetary Sciences, California Institute of Technology, Pasadena, CA 91125, USA \\ ${ }^{3}$ Space Science Institute, Macau University of Science and Technology, Macau \\ ${ }^{4}$ Division of Physics, Mathematics and Astronomy, California Institute of Technology, Pasadena, CA 91125, USA \\ ${ }^{5}$ Spitzer Science Center, California Institute of Technology, M/S 314-6, Pasadena, CA 91125, USA \\ Received 2014 July 9; accepted 2014 July 25; published 2014 August 6
}

\begin{abstract}
Asteroids of size larger than $150 \mathrm{~m}$ generally do not have rotation periods smaller than $2.2 \mathrm{hr}$. This spin cutoff is believed to be due to the gravitationally bound rubble-pile structures of the asteroids. Rotation with periods exceeding this critical value will cause asteroid breakup. Up until now, only one object, 2001 OE84, has been found to be an exception to this spin cutoff. We report the discovery of a new super-fast rotator, (335433) 2005 UW163, spinning with a period of $1.290 \mathrm{hr}$ and a light curve variation of $r^{\prime} \sim 0.8$ mag from the observations made at the P48 telescope and the P200 telescope of the Palomar Observatory. Its $H_{r^{\prime}}=17.69 \pm 0.27$ mag and multi-band colors (i.e., $g^{\prime}-r^{\prime}=0.68 \pm 0.03 \mathrm{mag}, r^{\prime}-i^{\prime}=0.19 \pm 0.02 \mathrm{mag}$ and SDSS $i-z=-0.45 \mathrm{mag}$ ) show it is a V-type asteroid with a diameter of $0.6+0.3 /-0.2 \mathrm{~km}$. This indicates (335433) $2005 \mathrm{UW} 163$ is a super-fast rotator beyond the regime of the small monolithic asteroid.
\end{abstract}

Key words: minor planets, asteroids: individual (335433) - surveys

Online-only material: color figures

\section{INTRODUCTION}

Asteroids, with the dwarf planet Ceres as the largest member, have sizes ranging from a few meters to a few hundred kilometers. They were subject to strong collisional interaction, as indicated by the formation of many asteroid families composed of breakup fragments from disruptive impact events. A fundamental discovery made by Harris (1996) concerning the internal structures of asteroids has to do with the statistical result that objects with sizes larger than $150 \mathrm{~m}$ have rotation periods longer than $2.2 \mathrm{hr}$. This spin cutoff can be explained by the rubble-pile structure in which the asteroids are made up of collisional breakup fragments bound together by mutual gravitational force. Faster rotation will lead to centrifugal disruption. Only one object, 2001 OE84, which is a near-Earth asteroid with a diameter of $0.65 \mathrm{~km}$ (Warner et al. 2009) and has a rotation period of 29.19 minutes, has been found to be exception to this rule (Pravec et al. 2002). Holsapple (2007) subsequently proposed that the binding of such fast rotating asteroids could be the result of size-dependent material strength and predicted the presence of kilometer-/sub-kilometer-sized super-fast rotators (hereafter SFRs). It is noteworthy to point out that several SFR candidates of this size range were reported by Masiero et al. (2009) and Dermawan et al. (2011), but none of them had reliably determined periods (Harris et al. 2012). This is because their low brightness and fast rotation make such SFRs difficult to be detected and confirmed by follow-up observations. A comprehensive program is required to produce quantitative measurements.

Within the framework of the $\mathrm{PTF} / \mathrm{iPTF}$ project $^{6}$ and the TANGO project, ${ }^{7}$ a systematic survey of asteroid light curves has been carried out by data mining of the archived data

\footnotetext{
6 The Palomar Transient Factory/intermediate Palomar Transient Factory: http://ptf.caltech.edu/iptf.

7 Taiwan New Generation OIR Astronomy.
}

(see Polishook et al. 2012 for the pilot study) and dedicated observational campaigns (see Chang et al. 2014 for the result for the first campaign) using the 48 inch Oschin Schmidt telescope (P48). In our second (2014 January 6-9) and third (2014 February 20-23) dedicated observational campaigns, a number of SFR candidates have been identified in the analysis of these data sets (the result including asteroid rotation periods and SFR candidates will be published in the near future). One of them is (335433) 2005 UW163. In this work, we report on the P48 discovery observation during 2014 February 20-23, and the follow-up observations conducted on the 200 inch Hale Telescope at the Palomar Observatory on 2014 March 25 to confirm the identity of this interesting object. In Section 2, we describe our observations and data reduction. The rotation period analysis is given in Section 3. The results and discussion are presented in Section 4. A summary and conclusion can be found in Section 5.

\section{OBSERVATIONS AND DATA REDUCTION}

\subsection{PTF Photometry}

The PTF is a synoptic survey designed to explore the transient and variable sky (Law et al. 2009; Rau et al. 2009). It employs the 48 inch Oschin Schmidt Telescope equipped with an 11 chip mosaic CCD camera that has a field of view of $\sim 7.26 \mathrm{deg}^{2}$ and a pixel scale of 1".01. The available filters include Mould $R$, Gunn $g^{\prime}$, and $H_{\alpha}$, and its $5 \sigma$ median limiting magnitude of an exposure of $60 \mathrm{~s}$ in the $R$ band is $\sim 21 \mathrm{mag}$ (Law et al. 2010). Each PTF exposure was processed by the IPAC-PTF photometric pipeline including image splitting, debiasing, flat-fielding, source extraction, astrometric calibration, and photometric calibration (Grillmair et al. 2010; Laher et al. 2014). Its final products include reduced images, mask images, and source catalogs. The PTF absolute photometric calibration was done by fitting to catalog data in Sloan Digital Sky Survey 


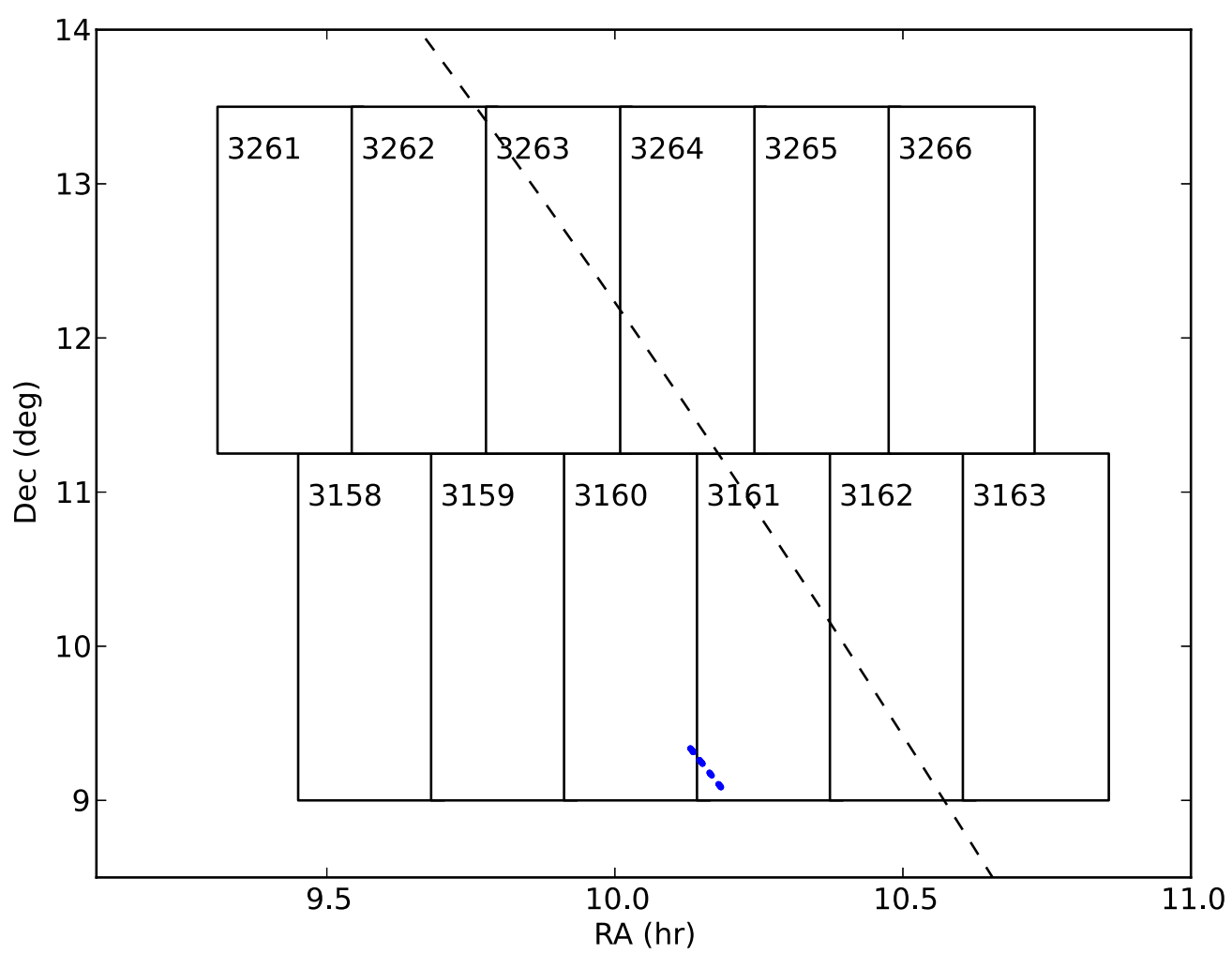

Figure 1. Configuration of 12 PTF fields. Each rectangle represents a PTF field with its field ID. The size of each PTF field is $\sim 7.26$ deg$^{2}$. The dashed line is the ecliptic plane and the blue dots are the trail of (335433) 2005 UW163. Note that the scales of R.A. and declination are not in the proper ratio.

(A color version of this figure is available in the online journal.)

Table 1

The PTF Observation

\begin{tabular}{|c|c|c|c|c|c|c|}
\hline Field ID & $\begin{array}{l}\text { R.A. } \\
\left({ }^{\circ}\right)\end{array}$ & $\begin{array}{c}\text { Decl. } \\
\left({ }^{\circ}\right)\end{array}$ & $\begin{array}{c}\text { Feb } 20 \\
\Delta t, N_{\exp }\end{array}$ & $\begin{array}{c}\text { Feb } 21 \\
\Delta t, N_{\exp }\end{array}$ & $\begin{array}{c}\text { Feb } 22 \\
\Delta t, N_{\exp }\end{array}$ & $\begin{array}{c}\text { Feb } 23 \\
\Delta t, N_{\exp }\end{array}$ \\
\hline 3158 & 143.65 & 10.12 & $7.9,19$ & $7.6,22$ & $7.9,22$ & $7.2,20$ \\
\hline 3159 & 147.12 & 10.12 & $8.0,17$ & $7.3,20$ & $7.9,21$ & $7.7,22$ \\
\hline 3160 & 150.58 & 10.12 & $7.6,18$ & $7.7,21$ & $7.7,22$ & $7.7,22$ \\
\hline 3161 & 154.04 & 10.12 & $8.0,20$ & $8.0,22$ & $7.9,23$ & $7.9,23$ \\
\hline 3162 & 157.50 & 10.12 & $8.3,20$ & $7.9,22$ & $8.0,24$ & $7.9,23$ \\
\hline 3163 & 160.96 & 10.12 & $8.0,19$ & $7.8,22$ & $8.0,24$ & $8.0,24$ \\
\hline 3261 & 141.55 & 12.38 & $7.7,20$ & $7.6,21$ & $8.2,23$ & $7.1,20$ \\
\hline 3262 & 145.05 & 12.38 & $8.1,20$ & $7.9,23$ & $8.2,23$ & $7.5,20$ \\
\hline 3263 & 148.54 & 12.38 & $8.2,20$ & $7.6,22$ & $8.1,23$ & $7.7,21$ \\
\hline 3264 & 152.04 & 12.38 & $7.9,20$ & $8.0,23$ & $8.2,24$ & $8.2,24$ \\
\hline 3265 & 155.53 & 12.38 & $8.7,19$ & $8.0,22$ & $8.1,24$ & $8.2,23$ \\
\hline 3266 & 159.03 & 12.38 & $8.0,21$ & $8.2,23$ & $8.1,25$ & $8.2,24$ \\
\hline
\end{tabular}

Notes. $\Delta t$ is the time duration spanned by each observing set in hours and $N_{\exp }$ is the total number of exposures for each night and field.

(SDSS) fields (York et al. 2000), which routinely reached a precision of $\sim 0.02 \mathrm{mag}$ (Ofek et al. 2012a, 2012b). Since the calibration was done on a per-night, per-field, per-filter, per-chip basis, small photometric zero-point variations are possible from night to night, the accuracy of which depends on the degree to which a night is affected by mist and cloud cover, and transient variations in atmospheric conditions.

As part of the TANGO project, we conducted an asteroid rotation-period survey during 2014 February 20-23, which continuously scanned 12 consecutive PTF fields on the ecliptic plane in $R$ band with a cadence of $\sim 20 \mathrm{~min}$ and an exposure time of $60 \mathrm{~s}$ for each frame. The total sky area was $\sim 87 \mathrm{deg}^{2}$. Table 1 lists the observational metadata and Figure 1 shows the field configuration. To extract known asteroid light curves, the source catalogs were purged of all stationary sources and then matched against the ephemerides obtained from the JPL/HORIZONS system with a radius of $2^{\prime \prime}$. In this campaign, we extracted $\sim 2500$ light curves suitable for rotation period analysis. The preliminary result showed $\sim 910$ objects had good rotation period determinations, and 11 of them were identified as SFR candidates. The result will be published in a separate paper soon. Follow-up observations for the SFR candidates are also planned. Our main-belt asteroid of interest, (335433) 2005 UW163, was close to its opposition and located on chips 7, 6, and 6 of field 3161 on 2014 February 20, 21, and 22, and on chip 11 of field 3160 on 2014 February 23. Therefore, we were able to obtain its light curve over a four-night time span to identify it as a highly probable SFR and conducted a follow-up observation to confirm its short rotation period.

\subsection{The Follow-up Observation}

The follow-up observation was carried out the night of 2014 March 25 using the 200 inch Hale Telescope at Palomar (hereafter P200), equipped for imaging with the Large Format Camera (LFC; Simcoe et al. 2000), which has a pixel size of 0.363 . In order to obtain the light curve and color of (335433) 2005 UW163, we followed an observational filter sequence of $2 g^{\prime}, 2 r^{\prime}, 2 i^{\prime}, 18 r^{\prime}, 2 g^{\prime}, 2 r^{\prime}, 2 i^{\prime}$, and $15 r^{\prime}$ bands. The time difference between two exposures was $\sim 3 \mathrm{~min}$, including a 2 min exposure time and a $\sim 1$ min readout time. Therefore, we obtained 47 exposures in total (i.e., $4 g^{\prime}, 4 i^{\prime}$, and $39 r^{\prime}$ bands) over a time span of $\sim 150$ minutes. We applied standard de-biasing, flat-fielding, and astrometric calibration to all images and used SExtractor (Bertin \& Arnouts 1996) to extract sources. The absolute photometry of each exposure was calibrated against SDSS point sources of $r^{\prime} \sim 18-22$ mag by 

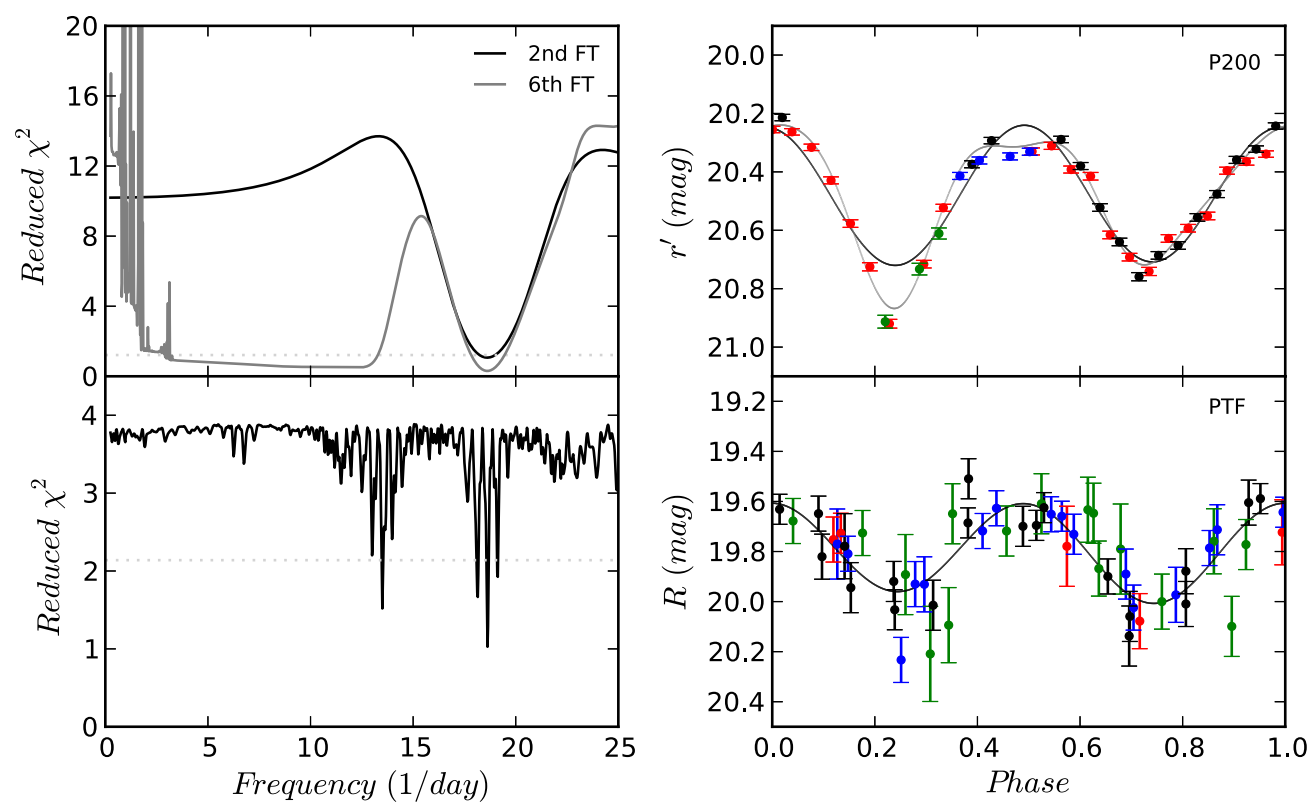

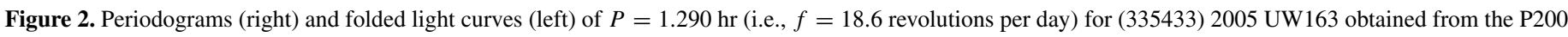

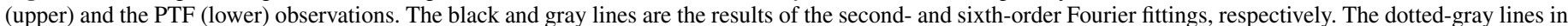

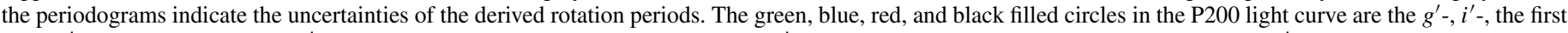

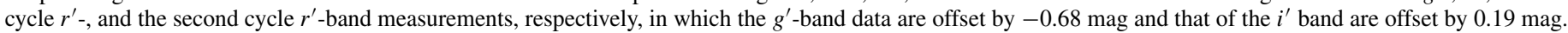
The green, red, blue, and black filled circles in the PTF light curve are the measurements taken on 2014 February 20, 21, 22, and 23, respectively.

(A color version of this figure is available in the online journal.)

using linear-least-square fitting and typically obtained a fitting residual of $\sim 0.02$ mag. Since (335433) 2005 UW163 would have a trail length of $\sim 2^{\prime \prime}$ for a 2 minute exposure, similar to the seeing size (i.e., $\sim 2^{\prime \prime}$ ) and much larger than the pixel size (i.e., 0'.363), we employed the trail-fitting method (Vereš et al. 2012) to improve its photometric accuracy. The first and last two exposures were not included in the following analysis due to their fuzzy images.

\section{ROTATION PERIOD ANALYSIS}

To measure the synodic rotation period of (335433) 2005 UW163, all data points were corrected for light-travel time (i.e., the time interval of a photon traveling from the object to Earth) and the magnitudes were reduced to both heliocentric $(r)$ and geocentric $\triangle$ distances at $1 \mathrm{AU}$ by

$$
M(r=1, \triangle=1)=m+5 \log (r \triangle),
$$

where $M$ and $m$ are the reduced and apparent magnitudes, respectively. The $r$ and $\triangle$ were calculated using PyEphem ${ }^{8}$ using the orbital elements obtained from the Minor Planet Center. ${ }^{9}$

We applied a second-order Fourier series, which was developed by Harris et al. (1989) and has since become the standard for asteroid light-curve fitting (e.g., Pravec et al. 2005), to the PTF $R$-band light curve and the P200 $r^{\prime}$-band light curve separately, in order to search for their rotation periods:

$$
\begin{aligned}
M_{i, j}= & \sum_{k=1,2}^{N_{k}} B_{k} \sin \left[\frac{2 \pi k}{P}\left(t_{j}-t_{0}\right)\right] \\
& +C_{k} \cos \left[\frac{2 \pi k}{P}\left(t_{j}-t_{0}\right)\right]+Z_{i},
\end{aligned}
$$

\footnotetext{
8 http://rhodesmill.org/pyephem/

9 http://minorplanetcenter.net
}

where $M_{i, j}$ is the reduced magnitude measured at the light-travel time corrected epoch $t_{j}, B_{k}$ and $C_{k}$ are the Fourier coefficients, $P$ is the rotation period, and $t_{0}$ is an arbitrary epoch. Following Polishook et al. (2012), we also fitted a constant value $Z_{i}$ in Equation (2) to correct the small systematic offsets between different PTF data sets, where a data set was defined as all the measurements taken on the same night, field, filter, and CCD. Then, Equation (2) was solved by least-squares minimization for each given $P$ to obtain the other free parameters. We tried a frequency range of $0.25-25$ revolutions per day with a step of 0.0025 revolutions per day to cover the majority of asteroid rotation periods (Pravec \& Harris 2000). The uncertainty of the derived rotation period was calculated from the range of periods with $\chi^{2}$ smaller than $\chi_{\text {best }}^{2}+\Delta \chi^{2}$, where $\chi_{\text {best }}^{2}$ is the $\chi^{2}$ of the picked-out period and $\Delta \chi^{2}$ is calculated from the inverse $\chi^{2}$ distribution, assuming $1+2 N_{k}+N_{i}$ degrees of freedom.

\section{RESULTS AND DISCUSSION}

The rotation periods of (335433) 2005 UW163 derived from the PTF and the P200 observations, which are $1.290 \pm 0.034$ and $1.290 \pm 0.058 \mathrm{hr}$, respectively, are very compatible with each other. The super-fast rotating nature of this asteroid is very convincingly demonstrated in both folded light curves (see Figure 2). The peak-to-peak variation of the PTF light curve is $R \sim 0.6$ mag and that of the P200 light curve is $r^{\prime} \sim 0.8$ mag. Although the P200 folded light curve does not cover two full cycles of the derived period, we do not see any particular offset in the overlap between the first (red filled circles) and second (black filled circles) cycles. Moreover, the light curve shows a large amplitude. Therefore, it is very unlikely that (335433) 2005 UW163 would be the case as discussed in Harris et al. (2014), where the authors pointed out that some asteroids might have ambiguous rotation period determinations due to their small light curve amplitudes (i.e., the half rotation period was misidentified as a "real" rotation period). One special case 


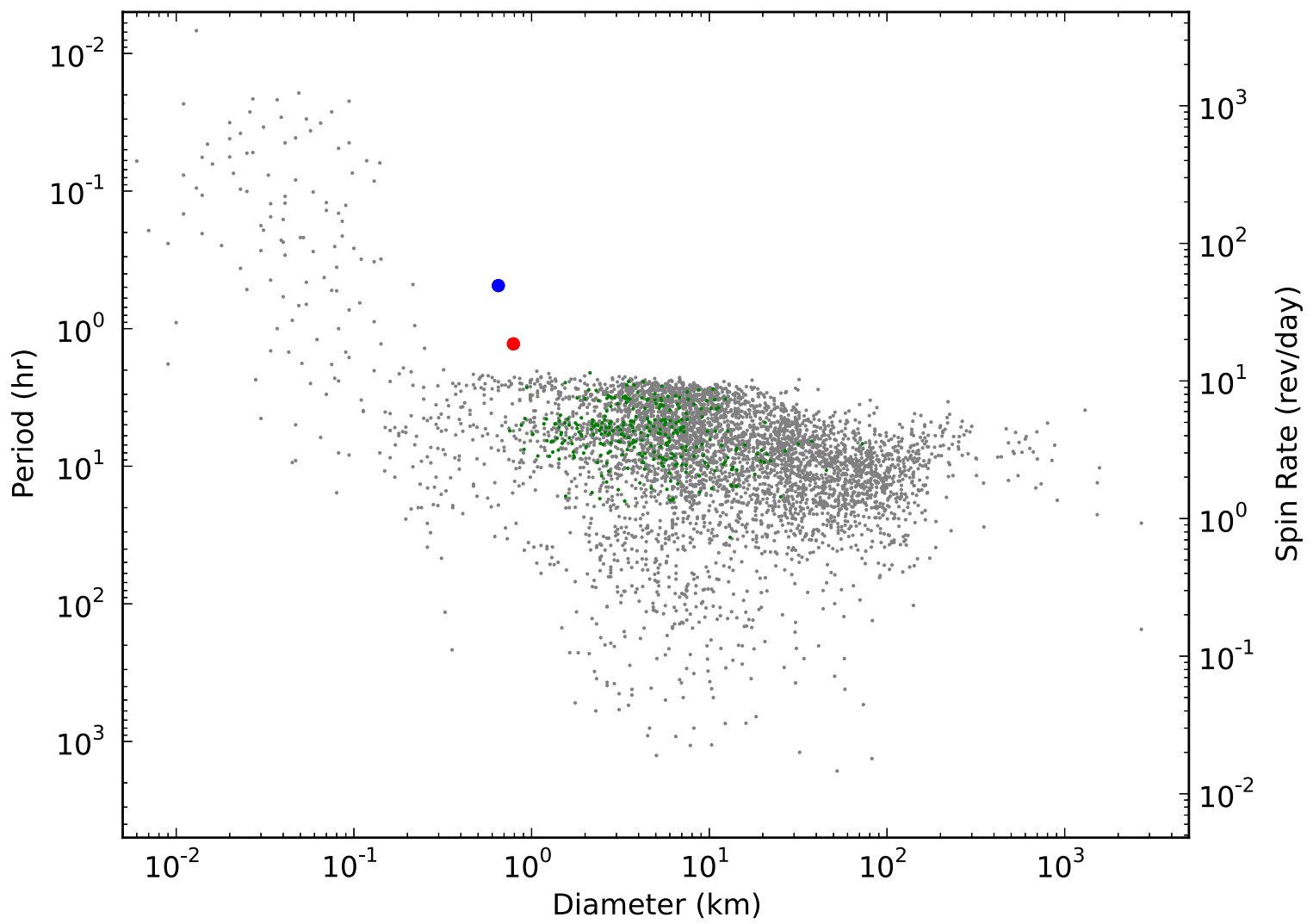

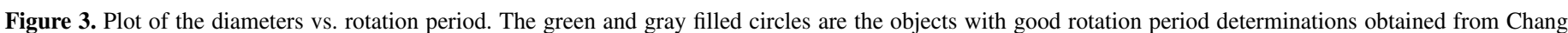

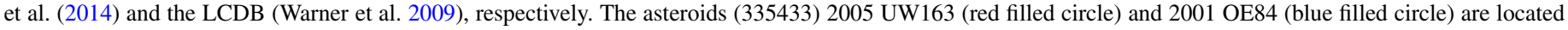
above the "spin barrier" and away from the small monolithic SFR.

(A color version of this figure is available in the online journal.)

reported by Polishook (2010) that (40701) 1999 RG235 showed a possible three-peaked light curve with a large amplitude. This could be a possibility for (335433) 2005 UW163. If the light curve of our target has four peaks, then it must have identical odd maxima, even maxima, and even minima as seen in our case. Such a chance seems to be very small, and we thus believe our derived rotation period is highly probable to be the true rotation period of (335433) 2005 UW163.

In order to improve the P200 light-curve fitting, we used a sixth-order Fourier series to analyze its rotation period again. The result is shown in Figure 2 as well. We see the best-fit rotation period still remains at $1.29 \mathrm{hr}$ and the fine features on the P200 folded light curve are reproduced relatively well as pointed out by Harris et al. (2014). This means (335433) 2005 UW163 has a more complex shape.

Since the $g^{\prime}$ and $i^{\prime}$ data points show similar variation to the P200 $r^{\prime}$-band light curve, we use Equation (2) with the derived rotation period, $1.290 \mathrm{hr}$, to obtain the offsets, $Z_{i}$, between the measurements for the $g^{\prime}, r^{\prime}$, and $i^{\prime}$ bands, where a data set was then defined as the measurements taken with the same filter. The best-fitting results were $g^{\prime}-r^{\prime}=0.68 \pm 0.03 \mathrm{mag}$ and $r^{\prime}-i^{\prime}=0.19 \pm 0.02 \mathrm{mag}$, which indicates an S- or V-type asteroid for (335433) 2005 UW163 (Ivezi'c et al. 2001). Incorporating $i-z=-0.45$ obtained from the SDSS moving object catalog (Ivezi'c et al. 2002), we believe (335433) 2005 UW163 is most likely a V-type asteroid (Parker et al. 2008).

Since the PTF and P200 light curves were observed in different bands and the phase angles $(\alpha)$ had a small change in both observations, we only applied, instead of fitting, the $H-G$ system (Equation (3) Bowell et al. 1989) with a fixed $G$ slope of $0.43 \pm 0.08$ (i.e., a typical $G$ value for a V-type asteroid; Warner et al. 2009) to the P200 $r^{\prime}$-band light curve to estimate the absolute magnitude $H$ of (335433) 2005 UW163.

$$
H=\langle M(r=1, \triangle=1)\rangle+2.5 \log \left[(1-G) \phi_{1}+G \phi_{2}\right],
$$

where

$$
\begin{aligned}
& \phi_{1}=\exp \left[-3.33 \tan (0.5\langle\alpha\rangle)^{0.63}\right], \\
& \phi_{2}=\exp \left[-1.87 \tan (0.5\langle\alpha\rangle)^{1.22}\right] .
\end{aligned}
$$

This gives a mean value of $H_{r^{\prime}}=17.69 \pm 0.27 \mathrm{mag}$. Moreover, its diameter is estimated using

$$
D=\frac{1130}{\sqrt{p_{r^{\prime}}}} 10^{-H_{r^{\prime}} / 5},
$$

where $p_{r^{\prime}}$ is the $r^{\prime}$-band albedo and the conversion constant, 1130, adopted from Jewitt et al. (2013). We assumed an albedo of $p_{r^{\prime}}=0.297 \pm 0.131$, which was adopted from the geometric albedo in visible wavelengths $\left(p_{v}\right)$ appropriate for a V-type asteroid (Usui et al. 2013). Although there is a small difference between $p_{r^{\prime}}$ and $p_{v}$, we treat this difference as part of the uncertainty in our assumed albedo. Therefore, we have a derived diameter of $D=0.6+0.3 /-0.2 \mathrm{~km}$, where the diameter range is estimated from the uncertainties introduced by $p_{r^{\prime}}, G$ and $H_{r^{\prime}}$.

Combining the data taken from Warner et al. (2009) and Chang et al. (2014), we plot the asteroid spin rate versus diameter in Figure 3. We see both (335433) 2005 UW163 and 2001 OE84 located above the "spin barrier" of the "rubblepile" asteroids and away from the small monolithic SFRs. 


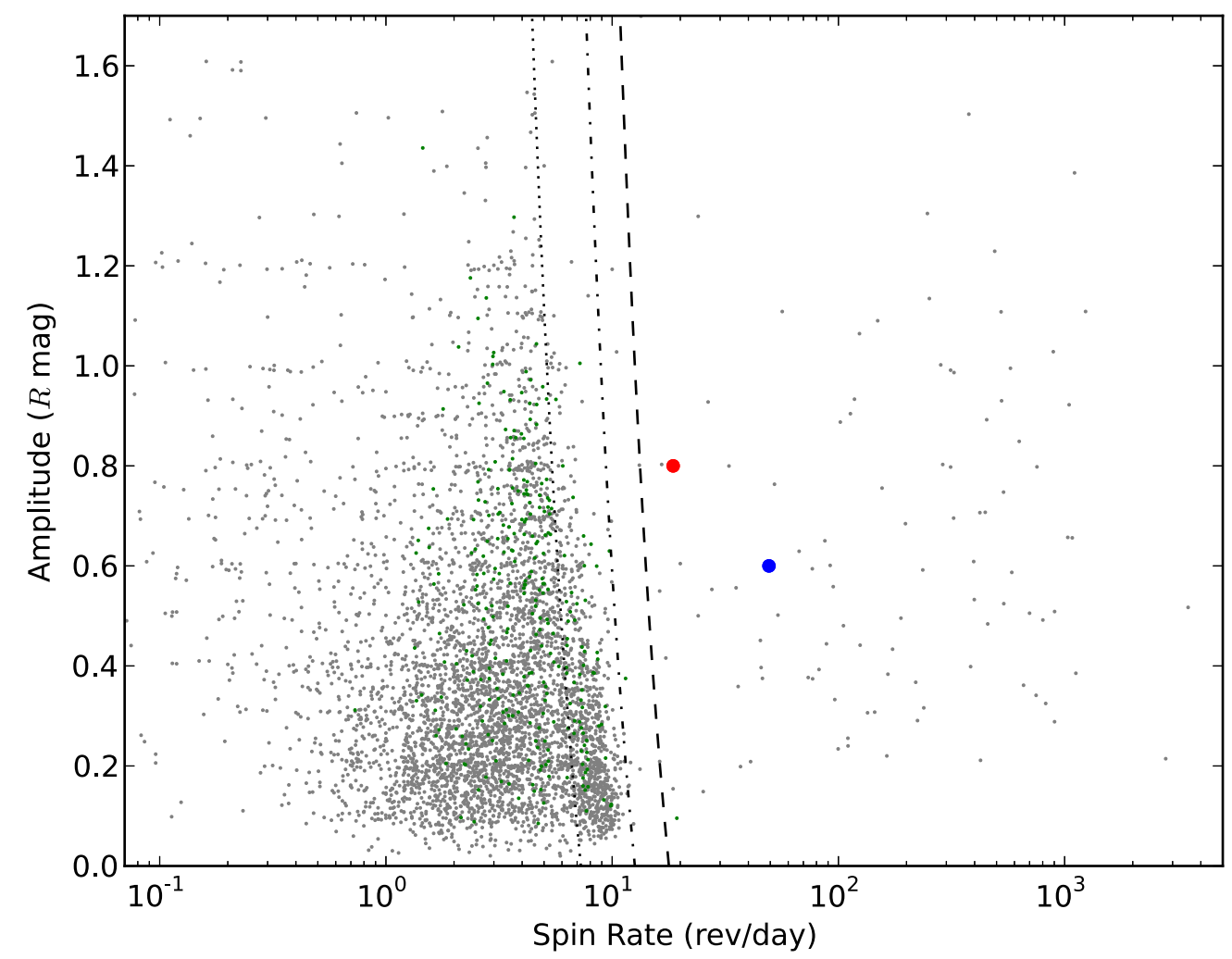

Figure 4. Plot of the spin rate vs. amplitude. The green and gray filled circles are the objects with good rotation period determinations obtained from Chang et al. (2014) and the LCDB (Warner et al. 2009), respectively. The dashed, dot-dashed, and dotted lines represent the spin rate limits estimated to be $P \sim 3.3 \sqrt{(1+\Delta m) / \rho}$ hr for "rubble-pile" asteroids with bulk densities of $\rho=6,3$, and $1 \mathrm{~g} \mathrm{~cm}^{-3}$ and light curve amplitude $\Delta m$ (Pravec \& Harris 2000). The asteroids (335433) 2005 UW163 (red filled circle) and 2001 OE84 (blue filled circle) have a bulk density larger than $6 \mathrm{~g} \mathrm{~cm}^{-3}$.

(A color version of this figure is available in the online journal.)

This result indicates (335433) 2005 UW163 is an SFR. To keep (335433) 2005 UW163 from breaking apart under such fast rotation by its own gravity only, it requires a bulk density as high as $\rho \sim 11 \mathrm{~g} \mathrm{~cm}^{-3}$ when assuming a critical rotation period of $P \sim 3.3 \sqrt{(1+\Delta m) / \rho}$ hr for an object with a light curve variation of $r^{\prime} \sim 0.8 \mathrm{mag}$ (Pravec \& Harris 2000). Such high bulk density is very unusual for the "rubble-pile" asteroids. This is because as shown in the plot of the spin rate versus light curve amplitude in Figure 4, the "rubble-pile" asteroids cannot have a bulk density $>3 \mathrm{~g} \mathrm{~cm}^{-3}$ (Pravec \& Harris 2000). To explain the presence of kilometer-/sub-kilometersized SFRs, Holsapple (2007) used a size-dependent strength for asteroids, which included tensile strength and cohesiveness, in addition to gravity. This additional size-dependent effect could produce a transition from the small monolithic object region to the large gravitationally bound aggregation region. Therefore, a comprehensive survey of the population of kilometer-/subkilometer-sized SFRs would help us understand this question.

\section{SUMMARY AND CONCLUSION}

The sub-kilometer-sized SFR (335433) 2005 UW163, which is a main-belt asteroid with a rotation period of $1.290 \mathrm{hr}$ (i.e., a frequency of 18.6 revolutions per day), was discovered in the asteroid rotation period survey conducted on the PTF during 2014 February 20-23 as part of the TANGO project. It was confirmed by the follow-up observation carried out on 2014 March 25 using the P200. Its multi-band colors (i.e., $g^{\prime}-r^{\prime}=0.68 \pm 0.03 \mathrm{mag}, r^{\prime}-i^{\prime}=0.19 \pm 0.02 \mathrm{mag}$, and the SDSS $i-z=-0.45$ ) indicate that (335433) 2005 UW163 is a V- type asteroid. With an assumed albedo of $0.297 \pm 0.131$, the SFR asteroid's $H_{r^{\prime}}=17.69 \pm 0.27$ mag allows us to infer a diameter of $D=0.6+0.3 /-0.2 \mathrm{~km}$. This shows that if (335433) 2005 UW163 is a "rubble-pile" asteroid, it would require a bulk density as high as $11 \mathrm{~g} \mathrm{~cm}^{-3}$ to keep itself from breaking apart under such fast rotation. Therefore, other mechanisms, such as a combination of tensile strength and cohesiveness, should be taken into account (Holsapple 2007). To search for a larger sample of kilometer-/sub-kilometer-sized SFRs through comprehensive asteroid rotation surveys, as is being planned, would be very helpful to the investigation of the origin and internal structures of asteroids.

This work is supported in part by the National Science Council of Taiwan under the grants NSC 101-2119-M-008-007MY3. We are greateful for the indispensable support provided by the staff of the Palomar Observatory. We also thank the referee, Alan Harris, for useful comments that helped to improve the content of the paper.

\section{REFERENCES}

Bertin, E., \& Arnouts, S. 1996, A\&AS, 117, 393

Bowell, E., Hapke, B., Domingue, D., et al. 1989, in Asteroids II, ed. R. P. Binzel, T. Gehrels, \& M. S. Matthews (Tucson, AZ: Univ. Arizona Press), 524

Chang, C.-K., Ip, W.-H., Lin, H.-W., et al. 2014, ApJ, 788, 17

Dermawan, B., Nakamura, T., \& Yoshida, F. 2011, PASJ, 63, 555

Grillmair, C. J., Laher, R., Surace, J., et al. 2010, in ASP Conf. Ser. 434, Astronomical Data Analysis Software and Systems XIX, ed. Y. Mizumoto, K.-I. Morita, \& M. Ohishi (San Francisco, CA: ASP), 28 
Harris, A. W. 1996, Lunar and Planetary Institute Science Conference Abstracts, 27,493

Harris, A. W., Pravec, P., Galád, A., et al. 2014, Icar, 235, 55

Harris, A. W., Pravec, P., \& Warner, B. D. 2012, Icar, 221, 226

Harris, A. W., Young, J. W., Bowell, E., et al. 1989, Icar, 77, 171

Holsapple, K. A. 2007, Icar, 187, 500

Ivezi'c, Ž., Tabachnik, S., Rafikov, R., et al. 2001, AJ, 122, 2749

Ivezi'c, Ž., Juric, M., Lupton, R. H., Tabachnik, S., \& Quinn, T. 2002, Proc. SPIE, 4836, 98

Jewitt, D., Ishiguro, M., \& Agarwal, J. 2013, ApJL, 764, L5

Laher, R. R., Surace, J., Grillmair, C. J., et al. 2014, arXiv:1404.1953

Law, N. M., Dekany, R. G., Rahmer, G., et al. 2010, Proc. SPIE, 7735, 3

Law, N. M., Kulkarni, S. R., Dekany, R. G., et al. 2009, PASP, 121, 1395

Masiero, J., Jedicke, R., Durech, J., et al. 2009, Icar, 204, 145

Ofek, E. O., Laher, R., Law, N., et al. 2012a, PASP, 124, 62
Ofek, E. O., Laher, R., Surace, J., et al. 2012b, PASP, 124, 854

Parker, A., Ivezi'c, Ž., Juri'c, M., et al. 2008, Icar, 198, 138

Polishook, D. 2010, MPBu, 37, 65

Polishook, D., Ofek, E. O., Waszczak, A., et al. 2012, MNRAS, 421, 2094

Pravec, P., \& Harris, A. W. 2000, Icar, 148, 12

Pravec, P., Kušnir'ak, P., Šarounov'a, L., et al. 2002, Aster. Comets Meteo., 500, 743

Pravec, P., Harris, A. W., Scheirich, P., et al. 2005, Icar, 173, 108

Rau, A., Kulkarni, S. R., Law, N. M., et al. 2009, PASP, 121, 1334

Simcoe, R. A., Metzger, M. R., Small, T. A., \& Araya, G. 2000, BAAS, 32,758

Usui, F., Kasuga, T., Hasegawa, S., et al. 2013, ApJ, 762, 56

Vereš, P., Jedicke, R., Denneau, L., et al. 2012, PASP, 124, 1197

Warner, B. D., Harris, A. W., \& Pravec, P. 2009, Icar, 202, 134

York, D. G., Adelman, J., Anderson, J. E., Jr., et al. 2000, AJ, 120, 1579 